\title{
Tecnologia digital de informação e comunicação como agente otimizador na relação ensino-aprendizagem na saúde
}

\author{
Digital information and communication technology as an optimizing agent in health teaching-lear- \\ ning relations \\ Información digital y tecnología de comunicación como agente optimizador en relaciones de enseñan- \\ za y aprendizaje de la salud
}

\section{Luciano Barbosa da Silva ${ }^{1 *}$, Cláudia Mara de Melo Tavares², Marilei de Melo Tavares ${ }^{3}$}

Como citar esse artigo. da Silva, LB; Tavares, CMM; Tavares, MM. Tecnologia digital de informação e comunicação como agente otimizador na relação ensino-aprendizagem na saúde. Revista Pró-UniverSUS. 2019 Jul./Dez.; 10 (2): 108-111.

\begin{abstract}
Resumo
Entende-se por TDIC (Tecnologias Digitais de Informação e Comunicação) o conjunto de dispositivos, plataformas, bases, ferramentas e ambientes que operem de forma digital processando dados e provendo informações tratadas e organizadas com modelos diversos de acessibilidade. Esta pesquisa traz à luz do conhecimento as implicações das utilizações das TDIC no processo formativo dos profissionais de saúde, identificando seus desafios, eficiências, entraves, perspectivas e tendências dentro do processo ensino-aprendizagem. Tem como objetivos: Provocar reflexões sobre experiências vivenciadas pelos profissionais de saúde apontando suas expectativas e dificuldades diante de um novo cenário de aprendizagem; identificar entraves na aplicação e utilização dos recursos digitais; analisar o processo de entrave das TDIC em sua inserção no cenário ensino-aprendizagem; discutir as TDIC à luz de referências teóricas contemporâneas do campo da educação; produzir um site que servirá como mapa apontando as principais plataformas TDICpara o ensino na saúde, disponibilizadas pelo Ministério da Saúde e seus parceiros. A metodologia definida para uso foi a pesquisa qualitativa, pois foram utilizadas informações com vínculo indissociável entre o profissional de saúde e os caminhos a ele apresentado para o aprendizado de conhecimentos inerentes a sua profissão, informações essas que não podem ser traduzidas em números. Foi escolhida uma abordagem sociopoética para a produção de dados junto ao grupo pesquisador. Os participantes do estudo são profissionais de saúde que estão realizando curso de pós-graduação stricto sensu (mestrado profissional, mestrado acadêmico e doutorado) na Escola Aurora de Afonso Costa/ Universidade Federal Fluminense - UFF. Também foi decidido realizar um levantamento de base bibliográfico e documental. Foi escolhido o software QSR Nvivo versão 10.0/Windows 64 bits para a análise qualitativa dos dados e geração de nuvem de palavras, bem como a organização das fontes de dados e geração de categorias. Também foi definido utilizaro aplicativo online wordle para produzir variações na nuvem de palavras junto com os resultados do QSR Nvivo. O respectivo projeto foi submetido à apreciação do Comitê de Ética em Pesquisa Humana, conforme definido na resolução CNS 466 sendo aprovado conforme parecer $n^{\circ} 3.628 .543$.
\end{abstract}

Palavras-chave: Informática, Saúde e Ensino.

\begin{abstract}
O Digital Information and Communication Technologies (TDIC) is the set of devices, platforms, bases, tools and environments that operate digitally processing data and providing information treated and organized with different accessibility models. This research brings to light the implications of the use of ICTs in the training process of health professionals, identifying their challenges, efficiencies, barriers, perspectives and trends within the teaching-learning process. Its objectives are: To provoke reflections on the experiences lived by health professionals pointing their expectations and difficulties in face of a new learning scenario; identify barriers to the application and use of digital resources; analyze the process of hindering ICTs in their insertion in the teaching-learning scenario; discuss ICDT in the light of contemporary theoretical references in the field of education; produce a website that will serve as a map outlining key TDIC platforms for health education. The methodology defined for use was the qualitative research, because information was used with inseparable link between the health professional and the paths presented to him for learning the knowledge inherent to his profession, information that cannot be translated into numbers. Devices from the Sociopoetic methodology were chosen for data production from the research group. The study participants are health professionals who are taking stricto sensu (professional master's degree, academic master's degree and doctorate) postgraduate courses at Aurora Afonso Costa School / Fluminense Federal University - UFF. It was also decided to conduct a bibliographic and documentary survey. QSR Nvivo version 10.0 / Windows 64-bit software will be used for qualitative data analysis and word cloud generation, as well as data source organization and category generation. It was also defined to use the wordle online application to produce word cloud variations along with QSR Nvivo results. The respective project was submitted for consideration by the Human Research Ethics Committee, as defined in resolution CNS 466 and approved pursuant to opinion 3,628,543.
\end{abstract}

Keywords: Computers, Health and Teaching.

Afiliação dos autores: 1. Graduado em Processamento de Dados. Mestrando do programa de Mestrado Profissional em Ensino na Saúde/MPES, UFF, RJ, Brasil. Email:professorinformatica@hotmail.com ORCID: https://orcid.org/0000-0002-3988-5614

2. Enfermeira. Professora Titular da Escola de Enfermagem -UFF. Doutora em Enfermagem. Brasil. Email: claudiamarauff@gmail.com ORCID: https://orcid.org/0000-0002-84166272

3. Psicóloga. Professora colaboradora do Programa de Mestrado Profissional em Ensino na Saúde/MPES, UFF, RJ, Brasil. Email: marileimts@hotmail.com ORCID: https://orcid. org/0000-0002-3276-0026

* Email de correspondencia: professorinformatica@hotmail.com 


\section{Resumen}

Tecnologías digitales de información y comunicación (TDIC) es el conjunto de dispositivos, plataformas, bases, herramientas y entornos que operan procesando datos digitalmente y proporcionando información tratada y organizada con diferentes modelos de accesibilidad. Esta investigación saca a la luz las implicaciones del uso de las TIC en el proceso de capacitación de los profesionales de la salud, identificando sus desafios, eficiencias, barreras, perspectivas y tendencias dentro del proceso de enseñanza-aprendizaje. Sus objetivos son: provocar reflexiones sobre las experiencias vividas por los profesionales de la salud señalando sus expectativas y dificultades ante un nuevo escenario de aprendizaje; identificar barreras para la aplicación y uso de recursos digitales; analizar el proceso de obstaculizar las TIC en su inserción en el escenario de enseñanza-aprendizaje; discutir ICDT a la luz de las referencias teóricas contemporáneas en el campo de la educación; producir un sitio web que sirva como un mapa que describa las plataformas TDIC clave para la educación sanitaria. La metodología definida para su uso fue la investigación cualitativa, porque la información se utilizó con un vínculo inseparable entre el profesional de la salud y los caminos que se le presentaron para aprender el conocimiento inherente a su profesión, información que no se puede traducir a números. Los dispositivos de la metodología sociopoética se eligieron para la producción de datos del grupo de investigación. Los participantes del estudio son profesionales de la salud que están tomando cursos de posgrado estrictos(maestría profesional, maestría académica y doctorado) en la Escuela Aurora Afonso Costa / Universidad Federal Fluminense - UFF. También se decidió realizar una encuesta bibliográfica y documental. El software QSR Nvivo versión 10.0 / Windows de 64 bits se utilizará para el análisis de datos cualitativos y la generación de nube de palabras, así como la organización de la fuente de datos y la generación de categorías. También se definió para usar la aplicación en línea wordle para producir variaciones de la nube de palabras junto con los resultados de QSR Nvivo. El proyecto respectivo fue sometido a consideración del Comité de Ética en Investigación Humana, tal como se define en la resolución CNS 466, y fue aprobado de conformidad con el dictamen 3.628.543.

Palabras clave: Informática, Salud y Docencia.

\section{Introdução}

O presente estudo trata a Tecnologia Digital de Informação e Comunicação (TDIC) e a sua relação no processo de ensino aprendizado na saúde com o propósito de evidenciar e suscitar reflexões sobre o tema através do ponto de vista da otimização dos recursos disponibilizados.

As características próprias da tecnologia, como: velocidade, armazenamento, duplicação, pesquisa e acesso vêm facilitando o envolvimento entre os sujeitos no processo de ensino e aprendizagem onde educando e educador devem estar em constante processo de capacitação teórica-prática, aprendendo e pesquisando o conhecimento base quanto às inovações tecnológicas digitais. Portanto percebe-se que o processo educativo em saúde aproveita frações deste universo de recursos nos fazendo repensar sobre o modelo atual de (des) preparo profissional digital neste novo cenário que se apresenta $^{1}$. A lei de Diretrizes e Bases da Educação (LDB) publicada em 1996 contempla uma plataforma de educação com características crítica e reflexiva apontando uma readaptação de aprendizagem conforme inserção das TDIC.

O objetivo geral desta pesquisa é analisar o uso de TDIC no processo ensino-aprendizagem em saúde disponibilizada pelo Ministério da Saúde e parceiros tendo em vista os desafios contemporâneos da educação. E como objetivos específicos, provocar reflexões sobre experiências vivenciadas pelos profissionais de saúde apontando suas expectativas e dificuldades diante de um novo cenário de aprendizagem; identificar entraves na aplicação e utilização dos recursos digitais; analisar o processo de entrave das TDIC em sua inserção no processo formativo do profissional de saúde; discutir as TDIC à luz de referências teóricas contemporâneas do campo da educação; elaborar um vídeo explicativo sobre o uso de TDIC para o ensino na saúde; produzir um site que servirá como mapa apontando as principais plataformas TDIC para o ensino na saúde desenvolvida pelo Ministério da Saúde e parceiros institucionais.

\section{Metodologia}

Foi definido realizar uma pesquisa qualitativa utilizando-se a Sociopoética² ${ }^{2}$. Os participantes são alunos pós-graduandos stricto sensu (mestrado acadêmico, mestrado profissional e doutorado) da Escola Aurora de Afonso Costa/Universidade Federal Fluminense - UFF. Em decorrência da abordagem sociopoética conceber uma construção coletiva de conhecimento, foi valorizado na pesquisa: pensar, conhecer, observar e aprender com o corpo inteiro dinamizando a razão pelas emoções e sensações. A Sociopoética é um caminho para o conhecimento que visa transformar os dados produzidos por uma pesquisa para melhor conhecer a realidade ${ }^{2}$. Tem como princípios norteadores ${ }^{8}$ : a) instituição do assim chamado "grupo pesquisador"; b) valorização das culturas populares e de resistência; c) a importância do corpo para a construção do imaginário, fazendo uso de todos os seus recursos como sensações, sensibilidades, intuições, emoções, razão, etc.; d) a importância do sentido espiritual, humano, das formas e dos conteúdos do saber; e) utilização de técnicas artísticas criativas para a produção de dados. Estas orientações que caracterizam a sociopoética vêm com a ideia de desconstruir a relação de poder entre pesquisador e sujeito, obstáculo que limita a pesquisa ${ }^{2}$. Sendo assim, o "pesquisador oficial" não coleta dados do seu grupo, ambos produzirão dados de "conteúdo consciente" e "conteúdo não consciente" num vai-e-vem do campo afetivo ao racional. E neste cenário, a arte se apresenta como dispositivo disparador da produção de dados. Os participantes do grupo pesquisador, que 
antes eram designados como sujeitos do grupo, agora são considerados copesquisadores influenciando a velocidade e rumo da produção chancelando a visão do pesquisador diante dos dados ou dando novos significados.

Então, para a produção de dados desta pesquisa, junto ao grupo pesquisador, houve dois momentos. No primeiro, um disparador artístico deu início à produção, trazendo a questão da utilização das TDIC como ferramentas de auxilio no processo ensinoaprendizagem bem como dispositivo de humanização na saúde. O disparador artístico foi composto por cenas pré-gravadas de voluntários interpretando três profissionais, a saber: um profissional de TI (tecnologia da informação), um engenheiro e uma enfermeira, interagindo de forma ensaiada e sincronizada com interpretações do pesquisador. Os profissionais das cenas pré-gravadas tentam persuadir o pesquisador a utilizar TDIC argumentando seus benefícios. Por fim, pesquisador resistente é persuadido, indo até a enfermaria de um hospital e faz uma "vídeo-chamada" para o parente de paciente que fazia semanas sem visita alguma. Paciente se emociona, agradece e o pesquisador corre para sua turma para compartilhar a utilização da TDIC. O grupo pesquisador, após observar a arte cênica, foi levado a refletir através da questão norteadora fazendo registro em formulários. Estes registros foram verbalizados numa roda de conversa. $\mathrm{O}$ grupo pesquisador também possuía outro bloco de anotações, chamado na sociopoética de "Diário de Itinerância" para livres anotações (emoções, intuição, afetos, etc) durante todo o encontro. O pesquisador oficial realizou gravação em áudio de todo o encontro, havendo, portanto três fontes de dados: diário de itinerância, registro das reflexões do disparador artístico e questão norteadora, gravação em áudio da roda de conversa. Após dispersão do grupo e nos dias seguintes, os dados foram transcritos, analisados, trabalhados e categorizados ${ }^{3}$ auxiliado pelo software Nvivo. Ao final a pesquisa estava pronta para a próxima etapa, que na abordagem sociopoética é chamada de contra análise. Esta fase é caracterizada pela apresentação das informações produzidas ao grupo pesquisador para que os dados sejam validados ou ajustados. Também foi utilizada arte criativa para esta etapa. Ao término da contra análise, o pesquisador possuía os dados necessários para realizar seus estudos e correlações com a revisão integrativa e com os atributos da própria pesquisa.Para o desenvolvimento desta pesquisa, foram atendidas as exigências da Resolução 466/12, do Conselho Nacional de Saúde, que é responsável pela normatização das pesquisas envolvendo seres humanos assegurando os preceitos éticos sendo submetido ao CEP/UFF (Comitê de Ética em Pesquisa) e aprovado conforme parecer $\mathrm{n}^{\mathrm{o}}$ 3.628.543. Na revisão integrativa, foi realizado um levantamento de base bibliográfico e documental tendo como base as publicações científicas indexadas na base de dados da Biblioteca Virtual em Saúde (BVS) e a Mendeleyentre 2013 e 2017. Foi escolhido o software QSR Nvivo versão 10.0/Windows 64 bits para a análise qualitativa dos dados e geração de nuvem de palavras, bem como a organização das fontes de dados e geração de categorias. Também foi definido utilizar o aplicativo online wordle para produzir variações na nuvem de palavras junto com os resultados do QSR Nvivo.

\section{Resultados Esperados}

Observando um novo cenário de aprendizagem desenhado com a participação das TDIC, podemos levantar três reflexões no campo da formação profissional: Evidências das TDIC na relação ensinoaprendizagem e saúde; Entraves no avanço da utilização das TDIC; Desafios para implementação das TDIC.

A educação em saúde vem dando espaço para as TDIC que vem trazendo consigo seus benefícios, seu dinamismo e força na propagação de conhecimento. As instituições, mesmo de forma tímida e com atraso, vêm utilizando estas tecnologias digitais em suas estratégias pedagógicas com uma grande variedade de aplicações. Outra forma de aprender, outra forma de comunicar vem sendo percebida como mais motivadora, interativa, ampla, democrática, transversal, multidisciplinar e sem limites geográficos ou de fuso horário evidenciando um instrumento eficaz para a formação, atualização e ampliação do processo formativo profissional em saúde ${ }^{7}$.

A motivação pelo conhecimento precisa ser articulada pela escola despertando o gosto e o prazer de aprender, proporcionando a capacidade de aprender a aprender ${ }^{4}$ ultrapassando as fronteiras da curiosidade intelectual propondo uma sociedade em que todos sejam professor e aluno alternadamente. Interessante refletir que as TDIC estão disponíveis e sendo utilizados pela maioria dos profissionais de saúde, isto é, a plataforma digital já é realidade, falta apenas adaptação para a realidade pedagógica. Uma pesquisa ${ }^{5}$ de 2014 realizada no Brasil, nos informa que $97 \%$ dos 72 profissionais analisados dominam e utilizam TDIC para suas tarefas particulares contra $35 \%$ para tarefas acadêmicas/ profissionais. Os desafios para implementação das TDIC merecem reflexões com observações no cenário de aprendizagem.

A manutenção de práticas pedagógicas orientadas pelo modelo tradicional de ensino vem sendo um dos maiores desafios para o avanço das TDIC no processo de ensino-aprendizagem na saúde. O conhecimento raso sobre a utilização e aplicação das tecnologias digitais para com o processo ensino-aprendizagem limita a ação dos gestores e docentes minimizando o avanço da produção e propagação de conhecimento. É necessário 
imaginação e criatividade para colocar em prática o leque de recursos destas tecnologias em prol do processo formativo dos profissionais. Há um impulso nos levando analisar um contexto mais abrangente trazendo ao palco do protagonismo cosmovisão, cultura e sentimentos que são itens relevantes para apresentação de caminhos alternativos para um aprendizado em linha com as novas tendências, em especial, as digitais ${ }^{5}$

Havendo as devidas motivações dos gestores, corpo discente e docente, as TDIC encontrarão seu espaço como coadjuvante neste grande palco do conhecimento chamado ensino-aprendizagem, trazendo seus benefícios de dinamismo, saber coletivo, compartilhamento, propagação e tantas outras características que somente as tecnologias podem tão bem realizar, afinal de contas, foi pra isso que elas surgiram. Inevitáveis e irreversíveis, as TDIC vieram para ficar, trazendo consigo suas mudanças, seus avanços, seus desafios e seu preço.

Vários portais ${ }^{6}$ já se consolidaram no meio acadêmico sendo utilizado por milhares de profissionais e usuários do SUS. Os profissionais de TI, os educadores, estudantes, profissionais e gestores da saúde precisam dar as mãos e mergulharem neste oceano de recursos que se apresenta fazendo uso de seus benefícios e desta forma propor qualidade ao atendimento dos usuários do SUS através de uma melhor formação de seus profissionais.

Entre a pedagogia conservadora e a contemporânea, as TDIC surgem como uma ponte ideal para esta transição. Que esta ponte seja alvo de investimentos, reflexões, atenções e construções para que o ganho de resultados seja de fato percebido para quem de direito é esperado: os usuários e profissionais do SUS.

\section{Implicações para relação ensino- aprendizagem na saúde}

Esta pesquisa vem mostrar a oferta e o potencial que tem as TDIC em proporcionar recursos que melhoram e dinamizam os procedimentos para formação profissional da saúde.

\section{Referências}

1. Leite KNS. A utilização das Tecnologias da Informação e Comunicação pelos docentes de enfermagem e as dificuldades no processo de ensino-aprendizagem. TEDE Sistema de Publicação Eletrônica de Teses e Dissertações da Universidade Federal da Paraíba. [publicação online]; 2014. Disponível em: <http://tede.biblioteca.ufpb.br:8080/handle/tede/5151>. Acesso em: 26 de setembro de 2017.

2. Tavares CMM. Como desenvolver experimentações estéticas para produção de dados na pesquisa sociopoética e abordagens afins? Revista Pró-UniverSUS. 2016 Jul./Dez.; 07 (3): 26-31

3. Bandin, L. Análise de Conteúdo. Trad. Reto, L; Pinheiro, A. São Paulo: Edições 70; 2011 .

4. Delors Jacques et al. Educação: um tesouro a descobrir. UNESCO;
Brasília, DF; ED.96/WS/9:2010. Disponível em: < https://unesdoc.unesco. org/ark:/48223/pf0000109590_por>. Acesso em:20 de outubro de 2019.

5. AlmeidaMM et al. O uso de tecnologias da informação e comunicação em áreas rurais é suficiente para a educação continuada?. J Bras Tele [revista em internet]. 2014; 3(1):211-219. Disponível em: <https://www.epublicacoes.uerj.br/index.php/jbtelessaude/article/view/10232>. Acesso em: 20 de outubro de 2018.

6. Tobase L et al. Recursos tecnológicos na educação em enfermagem. J. Health Inform. [revista em internet] 2013 Julho-Setembro. 5(3): 77-81. Disponível em <http://www.jhi-sbis.saude.ws/ojs-jhi/index.php/jhi-sbis/ article/view/218/172>. Acesso em: 26 de setembro de 2017.

7. Gama LN; Tavares CMM. Educação e mídia: implicações contemporâneas no cotidiano acadêmico. Texto \& contexto - enferm. [Revista em Internet]. 2015 junho; 24 (2): 593-599. Disponível em: $<$ http://www.scielo. $\mathrm{br} / \mathrm{scielo}$.php? script=sci_arttext\&pid=S0104-07072015000200593\&lng=en. http://dx.doi.org/10.1590/0104-07072015002052014>. Acesso em: 19 de outubro 2017.

8. Gauthier, J. Sociopoética e Formação do Pesquisador Integral. Revista Psicologia, Diversidade e Saúde. Salvador. 2015;4(1):78-86. Disponível em: $<$ https://www5.bahiana.edu.br/index.php/psicologia/article/view/459/526>. Acesso em: 29 de novembro 2019. 